\title{
Study Day of Palpable Masses
}

National Cancer Institute

\section{Source}

National Cancer Institute. Study Day of Palpable Masses. NCI Thesaurus. Code C119907.

The study day that a palpable masses assessment is performed. 